\title{
ALLE RADICI DELLA GRANDE CRISI. GIORGIO LUNGHINI E JOHN MAYNARD KEYNES
}

\author{
Nota del s.c. ANNA MARIA CARABELLI (*)
}

(Adunanza del 24 settembre 2020)

SuNTO. - Le note in ricordo di Giorgio Lunghini affrontano il suo approccio alla teoria economica e in particolare la sua analisi delle cause della grande crisi economica del 2007-2013. Egli identifica quattro cause principali della crisi: 1) la finanziarizzazione dell'economia; 2) il crescente indebitamento privato e pubblico; 3) gli squilibri strutturali commerciali e dei movimenti di capitale mondiali (in USA, Asia, sud America e in Europa). L'esistenza di paesi strutturalmente in deficit e in surplus commerciale, di paesi debitori e creditori; 4) il ruolo della teoria economica e in particolare il ritorno al laissez-faire/liberismo (monetarismo; critiche ai Keynesiani più che a Keynes che, secondo Lunghini, non è un Keynesiano «bastardo»); il ritorno alla "Treasury view", al "Washington consensus" e al "Bruxelles consensus". Nelle Note, queste quattro cause vengono analizzate in dettaglio.

$$
* * *
$$

ABSTRACT. - The notes in memory of Giorgio Lunghini deal with his approach to economic theory and in particular with his investigation of the causes of the great economic crisis of the 2007-2013 years. He identifies four main causes of the crisis: 1) the financialization of the world economy; 2) the growing debt of the private and public sectors; 3 ) the global trade and capital movement imbalances (in USA, Asia, South America and Europe); the existence of countries which are in a structural trade deficit and surplus; the sharp division between debtor and creditor countries; 4) the role of economic theory e in particular the return to the laissez-faire doctrine and liberalism (monetarism; the critiques to "bastard" Keynesians rather than Keynes who, in Lunghini's view, is not a Keynesian); the return to the "Treasury view", the "Washington consensus" and "Bruxelles consensus". In the Notes, these four causes are analysed in detail.

(*) Università del Piemonte Orientale, Italy. E-mail: anna.carabelli@uniupo.it 
Che cosa c'è, secondo Lunghini, alla radice della grande crisi, che è prima crisi dei prestiti sub-prime e poi crisi del debito europeo? Secondo lui, siamo di fronte a una fase - forse una fase terminale, ma non è detto - di trasformazione del capitalismo, cioè siamo di fronte a una delle molteplici forme in cui il capitalismo cambia il suo volto. Siamo di fronte a un capitalismo dominato dalla finanza. Questi sono sviluppi di lungo termine: ciò a cui assistiamo non è un fatto congiunturale; le sue radici hanno origini ben in là nei decenni scorsi. Sono sviluppi di lungo termine, che iniziano alla fine degli anni '60, proseguono nei decenni successivi e si manifestano sia nei paesi sviluppati sia nelle economie emergenti sia in quelle già emerse (perché molte di queste economie emergenti sono ormai emerse, ad esempio la Cina).

Quando Lunghini parla di un capitalismo dominato dalla finanza che cosa intende? Prima di tutto, questo capitalismo dominato dalla finanza è un capitalismo che ha già incorporato in sé la liberalizzazione e deregolamentazione dei mercati finanziari, in particolare del mercato monetario. Quest'ultimo è il mercato secondario dei titoli del debito pubblico. Nei decenni scorsi è avvenuta una liberalizzazione dei mercati finanziari e del settore bancario. Dietro a questa modificazione del funzionamento dei mercati c'è un mutamento teorico e la teoria che giustifica tale liberalizzazione e deregolamentazione dei mercati è, secondo Lunghini, e vi torneremo più avanti, la "teoria dei mercati efficienti”. Il secondo fattore molto importante è costituito dai mutamenti avvenuti nella legislazione e nella regolamentazione bancaria.

Facciamo un passo indietro e torniamo al 1933, all'altra grande crisi economica. Uno degli effetti della grande crisi del '29 è il fallimento sistematico delle banche. Nell'area europea, uno dei casi più emblematici fu il fallimento della banca tedesca Credit Anstalt, un fallimento che oggi tendiamo ad associare alla nascita del nazismo. Per fronteggiare la grande crisi del '29, la legislazione americana regolamenta il sistema bancario, introducendo il Glass-Steagall Act, una legge con la quale vengono separate le banche commerciali dalle banche di investimento (cioè la componente speculativa). Le banche commerciali, quelle dove depositiamo i nostri risparmi, vengono separate dalle banche di investimento, quelle che trattano i titoli finanziari. La legislazione del 1933 separa nettamente le banche commerciali dalle banche di investimento, chiarendo che le banche commerciali non possono essere banche di investimento: netta è la separazione di responsabilità e di competenza (non possono, per esempio, giocare con i nostri depositi). 
Durante gli anni ottanta del secolo scorso le lobbies, soprattutto bancarie e finanziarie, americane in primis, spingono sull'opinione pubblica e sul congresso americano per cercare di abolire questa legge perché impedisce loro di giocare su due tavoli contemporaneamente. Viene presa una delle prime decisioni significative da parte della Banca Centrale americana: il comitato comincia a discutere, e poi a votare, a favore di un alleggerimento della regolamentazione bancaria. Ci vogliono anni e molte pressioni delle lobbies, che continuano a lavorare e a muoversi su vari fronti, finché nel 1999 il congresso americano approva la "legge sulla modernizzazione dei servizi finanziari". Il GrammLeach-Bliley Financial Services Modernization Act di fatto cancella la legge del 1933 e riaccorpa le banche commerciali con quelle di investimento: le banche quindi possono passare facilmente da una all'altra funzione. Questo passaggio avviene sotto la presidenza di Clinton; quindi, ogni volta che vedete riapparire Clinton sulla scena insieme con Obama c'è da preoccuparsi. Obama, in seguito, cercherà di tornare indietro sul riaccorpamento delle banche con una nuova legge, che risulterà di fatto molto debole e molto fragile; le pressioni di Clinton e di Wall Street sono state determinanti.

Contemporaneamente, vengono attuate nuove strategie che in parte sono legate a questo cambiamento della legislazione bancaria. Sono mutamenti delle strategie delle banche commerciali, chiamate "originate-to-distribute". Le banche, cioè, creano nuovi strumenti finanziari - tornerò su questo più avanti per spiegare cos'è l'innovazione finanziaria - che poi distribuiscono ai mercati. Quali sono gli altri aspetti di questo capitalismo dominato dalla finanza? Alcuni sono notissimi. Innanzitutto le politiche di flessibilizzazione dei mercati, operate non solo sui mercati finanziari, ma anche su quello del lavoro e attuate in particolare negli anni ' 80 . Sono politiche estremamente pesanti di flessibilizzazione del mercato del lavoro, in parte Tatcheriane e in parte Reganiane. In Italia le abbiamo vissute un po' meno; le viviamo oggi in ritardo all'inseguimento di esperimenti esteri, provati da qualche altra parte e che, secondo alcuni economisti e politici, hanno dato buoni frutti. Quindi vengono attuate politiche di flessibilizzazione del mercato del lavoro, di deregolamentazione e mutamento della struttura istituzionale. Si verifica, in generale, una riduzione dell'intervento pubblico nell'economia di mercato. È un fenomeno chiarissimo se si considera l'Inghilterra. Il mutamento di questo paese negli anni '80 è lo specchio di un cambiamento sostanziale di quella società: chi ha vissuto 
in Inghilterra agli inizi degli anni '70, nel giro di pochissimi anni si trova di fronte a una società totalmente mutata dal punto di vista della sua struttura. Vengono attuate, in particolare, riduzioni delle politiche della domanda, cioè viene eliminata ogni politica di appoggio e sostegno della domanda aggregata; c'è una riduzione generalizzata del welfare state - la parola stessa "welfare state" diventa una parola negativa (proprio nell'Inghilterra di Beveridge, cioè delle conquiste del welfare state) -; viene privatizzata la sanità pubblica e il sistema pensionistico statale.

Il secondo mutamento, secondo Lunghini, riguarda i grandi cambiamenti nella distribuzione del reddito. Dagli anni '70 cresce la diseguaglianza nella distribuzione del reddito con uno spostamento dai salari verso i profitti, ma non tanto verso i profitti del settore industriale, ma verso le rendite finanziarie e in particolare immobiliari. Quei decenni sono caratterizzati da forti oscillazioni del prezzo degli immobili; si avvicendano grandi boom e crisi, il mercato immobiliare è molto tormentato, ma crescono le rendite.

L'altro aspetto che caratterizza questo capitalismo dominato dalla finanza è, secondo Lunghini, l'esplosione del debito. Oggi siamo consci di quali possano essere le dimensioni del debito pubblico, ma se guardiamo indietro vediamo come tale debito incomincia a crescere in modo notevole e come sia stato soprattutto un debito del settore privato, quindi in primis delle famiglie. Vi è un'esplosione del debito privato e in particolare del debito delle "classi povere" della società. Quindi una crescita del debito privato delle famiglie, in particolare di quelle povere. La crescita di questo debito - sebbene sia complesso spiegare un fenomeno di questo genere - è stata una crescita voluta e finalizzata a sostenere i consumi. Quando vi trovate di fronte a una società che ha salari troppo bassi, questi salari non permetterebbero di aumentare il consumo e di sostenere la domanda aggregata. Dovete tenere alti i consumi facendo indebitare i poveri. Il credito è soprattutto credito al consumo di beni durevoli, in primis crediti per la casa - che negli Stati Uniti è legata al "diritto di cittadinanza". Questo credito in seguito diventa credito al consumo generalizzato, in cui ogni acquisto o investimento avviene attraverso un forte indebitamento da parte del settore privato. Viene sostenuta la domanda, la produzione di beni e in particolare il settore edilizio che, in generale, è uno dei grandi moltiplicatori della produzione e del reddito. Va notato che la crisi dei prestiti sub-prime la miccia della crisi - è proprio una crisi provocata dall'insostenibilità dell'indebitamento privato. La crisi nasce dall'esplosione della bolla 
immobiliare; esplosione nel senso che i poveri non riescono a pagare $\mathrm{i}$ loro debiti.

Altro aspetto che caratterizza questo capitalismo dominato dalla finanza sono, secondo Lunghini, i fortissimi squilibri nella bilancia dei pagamenti, internazionali e intraeuropei. Dagli anni '70 si manifestano grandi squilibri commerciali, in particolare nel commercio tra nazioni e tra grandi aree economiche. Tali squilibri si manifestano su scala globale: tra gli Stati Uniti e l'area asiatica (la Cina soprattutto) e, a livello dell'area europea, tra quelli che vengono chiamati i "PIIGS" - cioè i "maiali", così vengono definiti dai mercati finanziari, Portogallo, Italia, Irlanda, Grecia e Spagna - e la Germania. Nell'area Euro, infatti, vi è una spaccatura a livello della bilancia dei pagamenti, della bilancia commerciale in particolare: da una parte vi sono i Paesi PIIGS e dall'altra la Germania. A livello mondiale, si manifestano quindi due enormi squilibri: uno a livello internazionale, che vede gli Stati Uniti da una parte e la Cina dall'altra, e uno a livello europeo (che è ciò che ci interessa maggiormente), che frappone i PIIGS e la Germania.

Per riassumere, quali sono, secondo Lunghini, le principali cause alla radice della crisi? La prima è di carattere teorico. Si sviluppa a partire dagli anni '70 e diventa visibilissima negli anni '80: un ritorno al liberismo attraverso forme diverse che chiamiamo "neoliberismo". In particolare, nella prima fase teorica si verifica un ritorno a ciò che viene chiamato "il monetarismo"; un'attenzione a Friedman e a Hayek che dominano il pensiero teorico in quegli anni, attraverso una forte critica a Keynes e ai keynesiani. Secondo Lunghini, la Teoria generale di Keynes è stata tradita:

"La soluzione del problema economico va dunque cercata altrove, non tanto nelle politiche 'keynesiane' illustrate nei manuali, magari mediante lo schema IS-LM, o invocate dai neoclassici temporaneamente convertiti, ma in un disegno di politica economica e sociale che si ispiri al capitolo 24 della Teoria generale, sulla Filosofia sociale verso la quale la Teoria generale potrebbe condurre" (G. Lunghini, La Teoria generale e i keynesiani: un'eredità giacente, Accademia Nazionale dei Lincei, Gli economisti post-keynesiani di Cambridge e l'Italia, 11 e 12 marzo 2009).

La seconda causa, secondo Lunghini, e lo abbiamo già visto, consiste in una finanziarizzazione dell'economia. Terzo fattore della crisi è l'enorme crescita del debito, che è una delle caratteristiche di questo capitalismo finanziario: un indebitamento privato, in prima battuta, che 
si trasforma poi in un indebitamento pubblico. Tutto ciò va sommato a squilibri strutturali di carattere commerciale: squilibri strutturali, non congiunturali; quindi, non modificabili nel breve periodo; enormi dal punto di vista della loro dimensione e associati a squilibri nei movimenti dei capitali internazionali. Si tratta di squilibri commerciali e nei movimenti di capitale internazionale che, in parte, sono associati a fenomeni di "globalizzazione". Gli squilibri portano a una divisione fra paesi in forte deficit e paesi in forte surplus commerciale, cioè una divisione del mondo in grandi debitori da una parte e grandi creditori dall'altra. Questo problema degli squilibri commerciali, che è meno noto e di cui si discute meno, è dal punto di vista teorico fondamentale per capire in quale situazione ci troviamo oggi. Va tenuto conto che vi è un doppio squilibrio: uno squilibrio mondiale e uno squilibrio interno all'area Euro; sono quindi, in generale, squilibri abbastanza complicati da analizzare. Abbiamo da una parte paesi che presentano un fortissimo surplus commerciale, cioè che esportano più di quanto importano; sono paesi esportatori, che hanno un eccesso di risparmio interno e che, per così dire, lo "buttano fuori" attraverso la loro bilancia dei pagamenti. Le nazioni in grande surplus sono, dal punto di vista teorico, paesi con un eccesso di risparmio. I creditori sono la Germania e la Cina. La Germania è un paese fortemente creditore, cioè in forte surplus rispetto al suo PIL, soprattutto se confrontato con il surplus cinese. In termini relativi, la Germania, come paese esportatore, batte la Cina. Le dimensioni del surplus della bilancia dei pagamenti tedesca sono enormi; come lo sono, dall'altra parte, in valore assoluto, le dimensioni del surplus commerciale cinese. Esistono, quindi, paesi con enorme surplus commerciale (i paesi creditori) e, dall'altra parte, paesi con fortissimo deficit commerciale (i debitori internazionali). Dal lato dei paesi debitori troviamo gli Stati Uniti (che è il più grande debitore internazionale) e i PIIGS. I "maiali” si trovano, perciò, dalla stessa parte degli Usa dal punto di vista dello squilibrio commerciale. L'altro aspetto su cui vorrei portare l'attenzione è che nei paesi debitori (questo vale sia per gli Stati Uniti sia per i cosiddetti "maiali") il consumo privato è sostenuto dall'indebitamento. L'indebitamento delle famiglie è, in parte, finanziato internazionalmente attraverso la bilancia dei pagamenti. A livello internazionale, i debitori si indebitano perché vengono finanziati dai paesi che stanno dall'altra parte del tavolo, cioè dai paesi creditori con un eccesso di risparmio. Ciò avviene attraverso i mercati finanziari internazionali. È chiaro quindi il ruolo della finanziarizzazione del sistema per 
sostenere il meccanismo di far indebitare i poveri all'interno di un paese e finanziarli attraverso i mercati internazionali. Mercati che nel frattempo sono stati liberalizzati per permettere ai capitali di muoversi da un punto all'altro del globo, come se si fosse in un solo mercato. Ma se i paesi debitori devono essere finanziati internazionalmente, questi paesi devono costantemente rivolgersi ai mercati per farsi finanziare. Di conseguenza, sono i mercati che decidono se finanziarli oppure no. Gli Stati Uniti sono obbligati a finanziarsi internazionalmente e questo finanziamento viene effettuato dalla Cina (cioè la Cina finanzia di fatto l'indebitamento delle famiglie americane attraverso i mercati internazionali). Gli Stati Uniti devono passare, e passano, attraverso questo meccanismo di mercato per finanziarsi, così come i PIIGS lo fanno all'interno dell'Europa. I debitori europei utilizzano il mercato monetario europeo. Potremmo porci la domanda: "i paesi europei possono finanziarsi anche da un'altra parte?”. Naturalmente sì, essendo i mercati globalizzati, se c'è qualcuno che è disposto a farlo, possono farlo. E lo fanno. Ma, di fatto, il finanziamento avviene prevalentemente all'interno dell'area europea.

La bilancia commerciale americana, agli inizi degli anni '80, comincia ad andare in rosso, cioè gli Stati Uniti diventano sempre più un paese debitore. Quando gli Stati Uniti entrano in crisi, cadono anche le loro importazioni, migliorando così il loro saldo commerciale; tuttavia, questo rimbalzo della bilancia commerciale americana è un segno ancor più visibile della loro debolezza, non di un riaggiustamento strutturale della loro bilancia dei pagamenti. Infatti, dopo il rimbalzo, la tendenza è di nuovo verso il basso. Lo squilibrio strutturale della bilancia dei pagamenti americana - del "grande debitore", come lo chiamiamo è uno dei grandi problemi internazionali irrisolti.

Se poi si analizza la bilancia dei pagamenti cinese dagli anni '90, perché la Cina precedentemente era completamente chiusa ai mercati internazionali - il surplus commerciale cinese, di fatto, è quasi tutto nei confronti degli Stati Uniti; la Cina è il "grande creditore" degli USA. L'aumento nel tempo diventa sempre più significativo. Le riserve in dollari della Cina, che di fatto sono rappresentate dai titoli americani in suo possesso, crescono; sono il finanziamento che la Cina fa nei confronti degli Stati Uniti. Questo vuol dire che la Cina detiene il debito americano e, ad esempio, quando Hillary Clinton andò in Cina, i cinesi le ricordarono che sono loro a detenere, finanziandolo, buona parte del debito americano. 
Allo stesso modo, se proviamo a leggere l'Eurozona e analizziamo il problema dello squilibrio commerciale europeo, possiamo vedere il contributo della Germania alla crescita europea. Se osserviamo bene, la Germania è il maggiore contributore alla crescita del PIL europeo; tutti gli altri paesi europei (tra cui ovviamente i PIIGS) contribuiscono in modo negativo. Nella crescita europea, la Germania è il paese determinante, tutti gli altri paesi sono una zavorra dal punto di vista della crescita europea. Inoltre è interessante osservare quanto la Germania, dal 2007-2008, domini in termini di contribuzione positiva, e quanto invece i PIIGS contribuiscano in modo negativo alla crescita europea. È possibile anche notare il peso, all'interno dell'Europa, delle due grandi aree: la Germania da una parte e i PIIGS dall'altra. Che cosa succede con la nascita dell'euro? Molti cominciano l'analisi prendendo a riferimento il 1999, quando l'euro è ancora virtuale ma l'euro entra in vigore pienamente solo nel 2002. Dalla nascita dell'euro, cresce lo squilibrio commerciale all'interno dell'Eurozona; ricordiamo che la Germania era già forte prima della nascita dell'euro. Quindi, la situazione non muta con l'euro; la moneta unica allarga ancor di più lo squilibrio europeo già esistente; cioè, si manifesta un crescente squilibrio commerciale all'interno dell'Eurozona. Ma come e perché si è verificato questo ulteriore crescente squilibrio? La risposta è facile: la Germania, dopo la riunificazione tedesca, persegue una politica di riduzione dei salari. L'obiezione potrebbe essere la seguente: "ma i salari tedeschi sono molto più alti dei nostri ...". Nel porsi questa domanda, però, non si valuta l'andamento dei salari in termini reali. La dinamica dei salari reali tedeschi viene rallentata dalla politica economica interna. Rispetto agli altri paesi europei, nell'ultimo decennio la Germania ha attuato una politica di riduzione dei salari. Questa politica, insieme all'adozione dell'euro, aiuta un paese fortemente esportatore. Va tenuto conto che l'euro è una moneta che, in generale, è sottovalutata rispetto a quello che sarebbe stato il marco tedesco; quindi, la Germania è stata avvantaggiata dall'avere l'euro rispetto al marco, perché il marco sarebbe oggi molto più forte dell'euro e penalizzerebbe le esportazioni tedesche. La Germania ha goduto di una competitività salariale e di una competitività di cambio nell'area esterna all'euro (non ovviamente in quella intra-europea) - cui si aggiunge una fortissima competitività di prodotto, dovuta all'innovazione dei prodotti tedeschi. Tutto ciò porta a crescenti surplus commerciali. Non è un caso che la Germania sia definita l'export Weltmeister, cioè il grande esportatore mondiale. 
Che cosa ha comportato la politica tedesca di contenimento dei salari? Che la domanda interna tedesca tendeva a scendere, proprio perché i salari domestici sono stati tenuti relativamente bassi. È stata una politica mirata alle esportazioni, a non aumentare i consumi interni: a produrre per cercare di vendere all'esterno. Quindi è stata legata a un contenimento della domanda interna tedesca e, comunque, a una riduzione della domanda di importazione da parte della Germania nei confronti, in particolare, degli altri Paesi UE, cioè dei "maiali". Più della metà del commercio estero della Germania avviene all'interno dell'Europa; quindi, il resto dell'Europa è rilevante per la Germania. Tali politiche tedesche non hanno soltanto contenuto i salari all'interno della Germania, ma, di fatto, hanno "bruciato" la crescita dei paesi deboli dell'Europa. A causa di questa politica tedesca, viene "bruciata" la crescita delle economie del Sud (ma non sono solo di quelle del Sud, perché c'è anche l'Irlanda in questo quadro), in un modo e in una misura tale che molte di queste economie non saranno in grado, nel futuro, di importare beni dalla Germania. Inevitabilmente, la Germania risentirà, come sta risentendo, della caduta delle importazioni da parte dei paesi deboli, come adesso risente del rallentamento della Cina. Quella tedesca è stata una politica mirata ad aumentare le proprie esportazioni, a scapito degli altri paesi europei.

Se consideriamo gli anni dopo la nascita dell'euro (cioè l'euro di fatto), noteremo che la forbice del commercio inter-europeo, cioè all'interno dell'Europa, fra la Germania e i paesi "maiali", si allarga. La Germania esporta verso i paesi deboli dell'Europa una quantità di prodotti elevata e importa da questi paesi la parte in basso che si vede nel grafico: nell'andamento della bilancia dei pagamenti tedesca è possibile notare una forbice che si allarga. Se osserviamo il periodo precedente, questa forbice era invertita. Nel periodo della riunificazione tedesca, la Germania, si finanziava e finanziava parte dell'allargamento ad Est attraverso le importazioni dal resto dell'Europa. In quel periodo era un'assorbitrice di risparmio. Appena dopo, se guardate il grafico, quando vien introdotto l'euro, il gap tra queste due aree ritorna ad essere "standard" della situazione tedesca: le esportazioni superano le importazioni.

Cerchiamo di delineare ora un altro quadro, per dare un'idea di quali siano le dimensioni del debito privato nel mondo; le famiglie sono fortemente indebitate. Ciò vuol dire che tutto il loro reddito viene speso e consumano di più del loro reddito; possiamo anche vedere le dimensioni del loro debito privato. Le famiglie del Canada, degli Stati 
Uniti, dell'Inghilterra, della Gran Bretagna, e di parte della Spagna sono fortemente indebitate. L'Italia non fa parte di questo gruppo: l'indebitamento privato nel nostro paese è, in termini relativi, minore. Invece l'indebitamento statale è più complicato da leggere. Il Giappone e l'Italia sono i "casi clinici" a livello internazionale, ma il Giappone sta peggio di noi in generale. Infatti, ha un indebitamento rispetto al PIL superiore all'Italia, cioè la sua situazione è di un debito pubblico completamente fuori controllo. Però questo debito è tutto detenuto dalle famiglie giapponesi. Quindi, per il Giappone, non si pongono i problemi di finanziamento internazionale che abbiamo descritto precedentemente. E per questo non rientra nei casi di squilibrio internazionale. Nel senso che la maggior parte del debito interno statale giapponese è detenuto dagli stessi giapponesi e non si pongono problemi di finanziamento esterno. Il caso dell'Italia è più travagliato, perché circa metà del suo debito era detenuto dall'estero. Adesso, però, la quota di titoli detenuta dall'estero è notevolmente scesa, anche a causa delle recenti enormi vendite dei titoli di stato italiani. Ma la cosa interessante, in questo grafico, e su cui voglio portare la vostra attenzione, è che nella crescita dell'indebitamento statale sono visibili i paesi in cui lo stato è intervenuto pesantemente nell'economia. Lo stato si è indebitato per salvare le banche private, con una fortissima crescita del debito statale. Tra questi paesi, la Gran Bretagna, di cui nessuno parla, ma che è uno dei grandi "casi clinici" nella situazione odierna; anche la Francia; e ovviamente gli Stati Uniti, in cui l'indebitamento statale è stato in parte alimentato dagli interventi di Obama, come gli hanno ricordato i Repubblicani. Tra questi, vi è anche la Germania, che è intervenuta pesantemente e, direttamente, per salvare le sue banche e che è intervenuta, in modo indiretto, con modificazioni della legislazione bancaria. Quindi, come vedete, siamo di fronte all'esistenza di un altissimo debito statale.

Se guardiamo alla crescente finanziarizzazione dell'economia mondiale e consideriamo l'economia americana perché è più facile da considerare, la finanziarizzazione è determinata dalla diminuzione del peso della manifattura rispetto ai servizi (in particolare, ai servizi finanziari) (i dati sono dal 1947 ad oggi) nel lungo periodo. La manifattura diminuisce il suo peso nella creazione del reddito. In parte questo fenomeno è dato dal mutamento della struttura produttiva di un paese - ed è giusto che scenda il peso della manifattura rispetto agli altri servizi ma qui è chiara una discesa molto significativa del peso del settore manifatturiero rispetto al resto dell'economia. I settori finanziari, del 
leasing, di tutte le assicurazioni, e la linea in giallo rappresenta il "settore strettamente finanziario". Da questi andamenti è ben evidente una tendenza di lungo periodo a processi di crescente finanziarizzazione dell'economia e di un peso sempre maggiore della finanza rispetto alla produzione industriale.

Quali sono, secondo Lunghini, le conseguenze della crescente finanziarizzazione? Questa finanziarizzazione è strettamente connessa ai mutamenti nella distribuzione del reddito. Di solito si tende a scindere questi due aspetti, mentre, secondo Lunghini, sono fortemente interconnessi: la finanziarizzazione dipende da mutamenti nella distribuzione del reddito ed è una risposta ai mutamenti nella distribuzione del reddito. Abbiamo visto che si verifica un aumento dei profitti rispetto ai salari, ma che, all'interno dei profitti, c'è un'ulteriore frattura fra la componente relativa alle rendite finanziarie vere e proprie rispetto ai "profitti industriali". Cambia il peso tra la finanza e il settore produttivo (il manifatturiero) e, la maggior parte di queste rendite finanziarie non ritornano al settore produttivo. Rimangono all'interno del settore finanziario e quindi accrescono maggiormente le rendite. Le dimensioni di tale componente puramente finanziaria tendono ad allargarsi sempre di più. In questo legame tra finanziarizzazione e distribuzione del reddito si allargano anche le disuguaglianze sia del reddito sia dei settori produttivi. C'è una crescita della disuguaglianza orizzontale tra finanza e industria. Il peso della finanza cresce; esplodono, in termini relativi, le remunerazioni della finanza rispetto a ciò che accade nel settore produttivo. E, contemporaneamente, si amplia la disuguaglianza verticale tra i salari dei dipendenti e la remunerazione dei top manager, soprattutto nella finanza. Arriviamo a rapporti spaventosi: a volte, 1 su 40, o 1 su 100 o anche di più. Questo accade sia nella finanza sia nelle banche. I top manager si appropriano di una quota sempre maggiore in termini di distribuzione del reddito. Un'altra caratteristica molto importante è che l'economia, in questo processo di finanziarizzazione crescente, sposta l'ottica dal lungo al breve e al brevissimo termine. Buona parte delle decisioni economiche sono tutte "miopi", cioè guardano molto vicino perché mirano a risultati di brevissimo termine. Mentre nel passato si era abituati a strategie di lungo termine - per esempio buona parte dello sviluppo dell'industria chimica tedesca dell'Ottocento derivava da strategie di lungo termine - con il processo di finanziarizzazione, l'ottica diventa di breve o di brevissimo termine. Un'ottica miope che però decide, di fatto, le strategie imprenditoriali 
del sistema produttivo. Accade che i mercati finanziari dettino le regole alla produzione e decidano le strategie imprenditoriali in un'ottica di brevissimo termine. La produzione dei beni vera e propria - e ciò che chiamiamo il "lavoro produttivo" in generale - escono di scena e non hanno più nessuna importanza economica. Sono i mercati finanziari a giudicare l'impresa e le sue strategie. Sono di nuovo i mercati a breve a giudicare anche il comportamento degli stati, perché i mercati rappresentano lo "standard" di valutazione.

Quali sono le altre conseguenze di questo processo di finanziarizzazione dell'economia? Avviene uno spostamento dell'attenzione dall'innovazione produttiva - cioè dai "processi di innovazione reale" - ai mutamenti interni alla finanza, cioè agli investimenti finanziari. Per darvi un'idea, molti di noi delegano la gestione del loro risparmio, magari anche piccole quote, a qualcun'altro. Il problema è che questo risparmio privato viene gestito attraverso strumenti finanziari, che non sono sotto il controllo diretto dei risparmiatori. Gli intermediari finanziari, e in molti casi la stessa speculazione "pura" che utilizza parte del risparmio privato delle famiglie, hanno un ruolo rilevante. Per cui, di nuovo, in questi processi di innovazione è più importante l'innovazione finanziaria di quella reale e produttiva. Come direbbe Schumpeter, l'idea stessa di innovazione reale esce di scena.

Torno nuovamente su un aspetto trascurato: la finanziarizzazione favorisce l'indebitamento. Se non ci fosse la finanza non ci sarebbe la possibilità di fare indebitare le famiglie. La possibilità di indebitarsi, quindi, è strettamente dipendente dall'esistenza di una struttura finanziaria e di una forte e crescente finanziarizzazione del sistema economico. Uno dei fenomeni che si è verificato nel periodo precedente alla recente crisi è la corsa a prestiti su garanzie inesistenti. Ho parlato all'inizio dei mutui sub-prime, cioè dei prestiti a persone, a famiglie in particolare, che si sapeva che non avrebbero mai potuto pagare i loro debiti (che diventano i "crediti inesigibili" per le banche). Se fate indebitare i poveri - e non è stato un processo casuale, come abbiamo visto - è noto che le loro garanzie sono inesistenti, o sono legate al prezzo degli immobili che è fortemente volatile. Quando cade il prezzo degli immobili i poveri non riescono più ad avere garanzie sufficienti per pagare $\mathrm{i}$ loro mutui e devono vendere la propria casa. Di conseguenza, per le banche, il loro credito diventa inesigibile.

Una delle caratteristiche dominanti in questo mutamento del capitalismo è, secondo Lunghini, la crescente fragilità finanziaria. Viene 
costruito un sistema che ha in sé le radici della sua distruzione o della sua implosione: era noto, infatti, che a un certo punto la bolla immobiliare e finanziaria sarebbe scoppiata. Precedentemente abbiamo visto che una delle caratteristiche della finanziarizzazione è la fortissima innovazione finanziaria, molto più rapida dell'innovazione reale. L'innovazione finanziaria è rapidissima, ma è anche imprevedibile, come cercherò di mostrarvi. Vengono inventati nuovi prodotti finanziari, alcuni incomprensibili anche agli stessi ideatori. Questi prodotti sono chiamati "strumenti finanziari complessi": nuovi strumenti derivati, costruzioni estremamente complicate dal punto di vista dei "pacchetti finanziari" che vengono costruiti.

Si sa, e si sapeva, che le garanzie sui prestiti a debitori insolvibili sono labili. Quando la banca presta a un debitore, che si sa insolvibile, cerca di "passare" questo rischio di insolvenza dal suo bilancio a qualcun altro, cerca cioè di "passare la patata bollente" per fare in modo che qualcun altro si scotti. Che cosa hanno fatto le banche? Hanno incentivato questi crediti inesigibili, li hanno poi passati ad altre istituzioni finanziarie, che li hanno impacchettati in strumenti finanziari complessi o "strutturati" - così vengono chiamati questi strumenti, ma chiamateli pure "pacchi bomba", che è il modo migliore di chiamarli. Li hanno passati dal bilancio delle banche ad altri operatori finanziari, che, a loro volta, li hanno passati ai risparmiatori, soprattutto a risparmiatori ignari. Quando il pacco bomba è esploso i risparmiatori si sono scottati. Questo è quello che è successo. Con tali strumenti complessi è stato, e viene ancora, nascosto il rischio sistemico. Molti di questi strumenti sono stati anche "passati" agli enti pubblici. Buona parte della trasformazione dei debiti degli enti pubblici in swap è stato effettuata con lo stesso meccanismo finanziario. Ignari (o forse ignoranti) amministratori pubblici hanno utilizzato questi strumenti finanziari, che sono poi scoppiati, lasciandoli (e ahimè, lasciandoci) con debiti spaventosi. Riprendendo l'idea del "passare la patata bollente" a qualcun altro, i crediti inesigibili vengono rivenduti a ignari risparmiatori: fino a che la catena di Sant'Antonio funziona, tutto va liscio; quando il debitore finale, cioè i poveri, non pagano, tutta la catena di Sant'Antonio salta. Quando la bomba della finanza innovativa scoppia, colpisce gli ignari risparmiatori che vengono in questo modo tosati. Il primo effetto è una caduta della ricchezza e del reddito dei risparmiatori, che hanno acquistato questi "pacchi bomba", il cui valore diventa pari a zero attraverso la caduta del prezzo delle quote dei fondi e delle attività finanziarie. Ma saltano anche 
gli intermediari finanziari. Quindi, salta l'anello a cui le banche avevano passato questi pacchi bomba: la Lehman Brothers è uno di questi intermediari. In quel caso, lo stato americano non è intervenuto a salvarla: l'hanno lasciata fallire per dare una lezione al mercato. Il risultato è stato di panico generalizzato. Oltre a questi intermediari finanziari, però, dovrebbero saltare anche le banche, perché alla fine i crediti inesigibili dovrebbe ritornare in mano loro. Nel caso delle banche, però, è intervenuto lo stato per salvarle; in molti casi, le banche sono state nazionalizzate. Alla fine, sono stati tosati i risparmiatori e, contemporaneamente, è stato fatto pagare ai cittadini il salvataggio del sistema bancario. Sono i cittadini che hanno pagato, e pagano. Oppure - come nel caso europeo - la Banca Centrale emette liquidità, che viene data alle banche per salvare le banche medesime e per evitare che falliscano.

Qui nasce un nuovo problema, su cui tornerò alla fine del mio contributo: l'idea che le banche siano "troppo grandi per fallire". Molte delle banche, o delle istituzioni finanziarie, sono troppo grandi. Quindi non vengono fatte fallire, come invece si dovrebbe fare; altrimenti coinvolgerebbero nel loro fallimento tutto il sistema creditizio e il sistema economico. Vorrei porre una domanda: la finanziarizzazione è, secondo Lunghini, una risposta alla crisi della crescita economica tra la fine degli anni '60 e l'inizio degli anni '70? C'è un legame tra la finanziarizzazione dell'economia e i timori di stagnazione economica della fine degli anni '60? La risposta è: “certamente, sì ...”. Come abbiamo visto, nell'evoluzione, o meglio nella trasformazione del capitalismo, la finanziarizzazione si sviluppa per far fronte al calo dei profitti nel settore privato, in particolare in quello produttivo. Greta Krippner, una sociologa dell'Università del Michigan, in un suo lavoro (Capitalizing on Crisis. The Political Origins of the Rise of Finance, Harward U.P. 2011), cerca di spiegare le radici politiche della nascita e della crescita della finanza e rintraccia - ovviamente studiando gli Stati Uniti, che sono il caso emblematico in questo processo di trasformazione - proprio nella finanziarizzazione la risposta alla crisi della crescita economica della fine degli anni '60. La finanziarizzazione è una risposta al mutamento della struttura del capitalismo alla fine di un processo di accumulazione, in cui viene lasciato al mercato, invece che alla politica, il ruolo di arbitro del conflitto distributivo. E qui ritorno al tema della distribuzione del reddito tanto caro a Lunghini. In tale processo di finanziarizzazione è il mercato a decidere come riallocare le quote distributive, che vanno a favore dei profitti e, in particolare, delle rendite. L'espansione 
del consumo, indispensabile nel processo di accumulazione capitalistica (altrimenti non avremmo avuto la produzione che abbiamo avuto), è avvenuta attraverso il mercato. L'aumento del credito ha fatto crescere l'economia e, alla fine, le attività finanziarie. Nella tradizione liberale, però, è il mercato che normalmente pone vincoli attraverso il sistema dei prezzi, cioè il prezzo di mercato è un fattore che pone limiti e regola. Questo non è avvenuto.

Il processo di finanziarizzazione ha promosso l'accesso al credito e ha creato un capitalismo in cui le attività finanziarie sono diventate sempre più rilevanti e redditizie. Uno dei maggiori promotori di questo processo è stato il governatore della Banca Centrale americana, Greenspan. Egli ha avuto un ruolo determinante in questo processo di accelerazione della finanziarizzazione. Va ricordato che egli è stato uno dei grandi fautori della liberalizzazione dei mercati e la sua politica ha favorito il laissez-faire del mercato, cioè il lasciare al mercato le decisioni e il ruolo di arbitro generale.

Sono gli speculatori a provocare la crisi? Gli speculatori giocano in un ambiente che è creato per loro e, ovviamente, se c'è un sistema che favorisce il gioco, loro giocano. C'è certamente una componente di euforia irrazionale nella crescita della speculazione, ma questa crisi, come ho cercato di mostrare, è generata da processi di lungo corso, da trasformazioni molto precise nel modello di crescita economica e, certamente, è legata a una rinuncia da parte della politica al suo ruolo di regolatore generale. Questo è uno dei punti fondamentali: la politica recede ed è il mercato che si occupa di regolare questi processi e, ovviamente, la distribuzione del reddito.

A che punto siamo arrivati nel processo di finanziarizzazione dell'economia? Allo scoppio della crisi siamo arrivati a un punto in cui la finanziarizzazione ha raggiunto livelli di ingovernabilità; quindi c'è un problema di come far rientrare il genio dentro alla lampada, ma è difficile rimandarlo al suo interno. La politica ha certamente abdicato al suo ruolo, a una parte essenziale del suo compito, forse anche per incapacità. È un problema complicato anche solo valutare se è stata incapacità o se sono state prese volontariamente decisioni per dare risposte concrete alle difficoltà della crescita. A tale proposito, qual è stata la contrapposizione teorica che ha permesso tutto questo? Ho già accennato, all'inizio, che, secondo Lunghini, esiste un conflitto teorico; a un certo punto, negli anni ' 70 , si è verificata una rinascita della teoria liberale (neoliberale), alimentata da una forte critica teorica. C'è stata, 
infatti, una contrapposizione tra quella che si può chiamare la "teoria dominante" e la "teoria di Keynes" (per Lunghini, Keynes non è un keynesiano bastardo, così che si devono distinguere queste due componenti). Qual è lo scontro teorico tra queste due teorie? In Keynes sono presenti tre mercati:

- il mercato della moneta;

- il mercato dei beni;

- il mercato del lavoro.

Il mercato della moneta è ciò che chiamiamo, appunto, il mercato monetario (la finanza in breve). Il mercato dei beni è dove vengono scambiate le merci; ciò che Keynes chiama la domanda e l'offerta aggregata. Il mercato del lavoro è quello in cui si ha l'offerta di lavoro da parte dei lavoratori e la domanda di lavoro da parte delle imprese. Se prendiamo in considerazione questi tre mercati, nella teoria dominante, vengono rappresentati come degli "orti chiusi”, cioè ciascun mercato è separato dagli altri. Quindi, i mercati non comunicano l'uno con l'altro; nella teoria dominante, la rappresentazione schematica è quella di avere tre mercati indipendenti, separati l'un dall'altro. Nella teoria dominante, che cosa accade quando si manifesta, per esempio, una crisi all'interno del mercato della moneta? Dal punto di vista teorico, lo squilibrio rimane interno al mercato della moneta; non "schizza teoricamente" niente sugli altri mercati. E' uno squilibrio interno a un "orto ben recintato". Una crisi di carattere monetario-finanziario rimane all'interno di quel mercato e, dal punto di vista teorico, non ha alcun effetto sugli altri mercati. Ciò avviene attraverso meccanismi teorici abbastanza raffinati, che chiamiamo di demarcazione, di separazione fra il mercato della moneta e i mercati reali. Cosa succede invece secondo la teoria di Keynes? Per Keynes, esistono mercati gerarchici - immaginate un condominio a più piani: il mercato della moneta è il piano più importante, quello più in alto rispetto agli altri piani. Gli altri mercati sono gerarchicamente, dal punto di vista teorico, mercati dominati, cioè stanno al di sotto del piano della moneta. Immaginiamo di avere delle scatole una sopra l'altra: il mercato della moneta è quello più in alto, come se fosse il piano più in alto dove c'è anche un grande terrazzo, poi sotto, c'è il mercato dei beni e, per ultimo, quello del lavoro, che sta al piano terreno (ad esempio, la portineria). Ciò che succede ai piani alti della teoria - cioè nel mercato della moneta - ha effetti, attraverso le variabili teo- 
riche, sui mercati che stanno in basso: in Keynes è il livello del tasso di interesse che trasmette i suoi effetti ai mercati sottostanti, al punto che "percola" nel mercato dei beni e successivamente in quello del lavoro. Il mercato del lavoro, secondo Keynes, è un mercato finale, un mercato passivo, su cui si scaricano tutti gli aggiustamenti che avvengono ai piani alti dei mercati. Quando si verifica un problema al livello del terrazzo, ad esempio una perdita d'acqua, quelli che stanno sotto ne subiscono le conseguenze. E, in Keynes, il mercato del lavoro non ha alcuna capacità di influenzare, direttamente, i mercati che stanno al di sopra. Quindi, quando si parla di disoccupazione, la disoccupazione può anche dipendere dal non perfetto funzionamento della portineria, cioè del mercato del lavoro. Ma, se non si sta attenti alla struttura teoricagerarchica generale dei mercati non si riesce a capire che cosa causa la veramente la disoccupazione. Keynes spiega la disoccupazione con il malfunzionamento dei mercati che stanno, dal punto di vista teorico, al di sopra del mercato del lavoro. Per Keynes, i mercati non sono "orti chiusi": c'è un mercato dominante, quello della moneta, e questo mercato scarica i suoi effetti su quello immediatamente sottostante, che è il mercato dei beni; il quale, a sua volta, influenza il mercato del lavoro.

Nella discussione teorica tra keynesismo bastardo e monetarismo (neoliberismo) degli anni '70, Keynes perde la battaglia. La teoria keynesiana viene eliminata e ridiventa dominante la teoria che Keynes stesso aveva combattuto nel 1936. Ho precedentemente accennato che parte della spiegazione sulla liberalizzazione deriva da una specifica teoria del mercato della moneta, quella dei mercati efficienti. La discussione teorica degli anni ' 70 anticipa ciò che poi avviene più tardi nelle politiche economiche e nei mutamenti istituzionali, - processi che sono ovviamente più lenti di quelli teorici. Secondo la "teoria dei mercati efficienti", proposta da Eugene Fama in un suo noto lavoro del 1970, nel mercato della moneta - ricordate che è "un orto chiuso" per la teoria dominante - i prezzi dei titoli riflettono tutte le informazioni esistenti. E' quindi un mercato perfetto, perfettamente efficiente, dove gli agenti sono perfettamente razionali e dotati di conoscenza perfetta e di onniscienza, direbbe qualcuno. Per Keynes, invece, nel mercato della moneta c'è conoscenza limitata. Ricordo che Keynes è un grande studioso di probabilità; ha scritto un testo molto importante proprio sul problema della conoscenza limitata. In Keynes, la conoscenza - soprattutto nel mercato della moneta, ma anche in quello dei beni - è limitata ed è, in molti casi, dominata dall'ignoranza. C'è quindi un aspetto 
cognitivo fondamentale di contrapposizione delle due teorie. Scrive Lunghini,

"In letteratura, e soprattutto nei libri di testo, spesso si discute di quale sia la vera innovazione analitica presente nella Teoria generale: se l'innovazione consista nel principio della domanda effettiva, oppure nella determinazione monetaria del tasso di interesse. La discussione è oziosa, poiché entrambe le categorie, e dunque l'intera Teoria generale, piaccia o non piaccia, e ai più non piace, hanno una fondazione comune, che per brevità e provvisoriamente si può definire di ordine psicologico. Un approccio, questo, guardato con sospetto non soltanto dagli economisti neoclassici cui si indirizza la critica keynesiana, ma anche da molti economisti keynesiani e post-keynesiani. Gli economisti - in generale - preferiscono il ragionamento deduttivo e deterministico" (G. Lunghini, La Teoria generale e $i$ keynesiani: un'eredità giacente, Accademia nazionale dei Lincei, Gli economisti post-keynesiani di Cambridge e l'Italia, 11 e 12 marzo 2009).

Nella crisi c'è stata una crisi di insolvenza e una crisi di liquidità da parte delle banche. Ora vorrei concentrarmi sulla crisi di liquidità da parte dalle banche europee. Questo avviene quando le banche trattengono la liquidità, non la "passano", per esempio, alle imprese. Come abbiamo visto, nella teoria di Keynes il mercato della moneta è un mercato gerarchico, domina gli altri mercati, quindi è fondamentale. Keynes spiega la speculazione in modo behavioristico: chi opera sui mercati monetari-finanziari non agisce in base a decisioni perfettamente razionali, ma attraverso "la psicologia di massa". Nella teoria dei mercati efficienti, invece, poiché si ritiene che gli operatori nei mercati monetari-finanziari siano efficienti, allora non bisogna intervenire. Anzi, se si interviene si cambiano i segnali del mercato, cioè i prezzi. L'unica cosa è laissez-faire (lasciar fare al mercato). Non si deve intervenire, non si deve regolamentare i mercati e, anzi, se li avete regolamentati dovete deregolamentarli, perché i mercati si autoregolano e funzionano meglio.

Keynes scrive La Teoria Generale nel 1936 e ha davanti a sé gli effetti della grande recessione che segue la crisi del'29. Nel capitolo 12, afferma che: "gli speculatori possono non causare alcun male, se sono pochi", però, "quando lo sviluppo del capitale - qui parla delle trasformazioni del capitalismo - diventa il sottoprodotto delle attività di un casinò - in cui domina la speculazione rispetto ad altre attività produt- 
tive - è probabile che ci sia qualcosa che non va" (ora sappiamo perché). Sempre nel capitolo 12, prosegue: "come i casinò devono essere nel pubblico interesse inaccessibili e costosi" - e qui c'è un parallelo con la Tobin Tax anche se, ahimè, la Tobin Tax verrà fatta pagare ai risparmiatori, e non la pagheranno le banche e gli intermediari. Scrive sempre Keynes: "questo è inaccessibile e costoso...". Questo passo si riferisce ai mercati finanziari. La sua idea è che l'accesso al casinò debba essere reso difficile e regolamentato. Ci sono passi nella Teoria Generale in cui Keynes afferma che bisogna limitare il ruolo della finanza. L'idea è rivoluzionaria, è anche un po' drastica. E'un'idea che non vince in un mondo dominato dalla finanza.

Abbiamo visto che, secondo Lunghini, la crisi è anche il risultato di una sconfitta teorica; Keynes (o meglio i Keynesiani bastardi) viene sconfitto dal pensiero neoliberale negli anni ' 70 . La finanziarizzazione dell'economia è anche spiegata teoricamente da tale sconfitta. La crisi, causata dalla finanza, dovrebbe quindi essere interpretata come una crisi del "neoliberismo". Dal punto di vista teorico e del controllo dell'ideologia dominante, tuttavia, la finanza non si è assunta - e non assume - alcuna responsabilità della crisi. La teoria economica rimane invece saldamente ancorata ai suoi principi neoliberali. Keynes, che è stato resuscitato per un po' durante i momenti più bui della crisi, esce definitivamente di scena. Ora - e la cosa è rilevante - la responsabilità della crisi viene addossata allo stato e non alla finanza. Si parla della crisi del debito sovrano: è quindi lo stato - e non la finanza o le banche - il colpevole della crisi. Lo stato ha accumulato troppo debito pubblico; le politiche economiche perseguite dallo stato, per sfuggire alla crisi e sostenere la crescita economica, sono criticate in quanto misure keynesiane. Monti continua a ripetere, e non è l'unico: "non è attraverso Keynes... non è attraverso le misure keynesiane, che noi ne usciremo". "Austerità" è il motto; l'austerità ci salverà. Questo significa tagli alla spesa e alla domanda, tutte misure restrittive. Anche quando si parla di crescita, la crescita viene difesa solo con manovre sull'offerta, che non sono manovre keynesiane. Sono legate alla teoria neoliberale. Queste manovre sull'offerta sono: riduzione dei prezzi, riduzione dei salari, miglioramento della competitività, aumenti della produttività, aumenti della concorrenza. E quando Monti parla di manovre sull'offerta, propongono tutte misure di crescita neoliberale. Ma, che cosa sono queste politiche di austerità che mirano alla crescita? L'idea fondamentale è che tutti i paesi devono adottare politiche che gli economisti riassumo- 
no con la frase "cercare di fregare il proprio vicino". Cioè, ogni paese dovrebbe - attenzione al condizionale - cercare di esportare di più a scapito di qualcun altro: quindi, si tratta di politiche per spingere le proprie esportazioni. Tali politiche si chiamano in generale "politiche neomercantiliste" (e quindi non sono per niente neoliberali) e il modello tedesco è lo standard: la Germania è infatti la grande neo-mercantilista, adotta la politica mercantilista di aumentare le esportazioni e il surplus, come abbiamo già visto. In questa crisi, il problema complicato è che se tutti cercano di fregare il proprio vicino, come direbbe Keynes, ne risulta un effetto di "fallacia di composizione", in cui nessuno riesce più a esportare e tutti si avvitano in un processo recessivo, come sta avvenendo. Quindi, se tutti tagliano la domanda (austerità), in particolare la domanda interna, e tutti cercano di esportare di più, chi consuma e chi importa? In passato, in questo gioco "compositivo" il grande importatore - noi economisti lo chiamiamo, con una battuta, "l'importatore di ultima istanza" - erano gli Stati Uniti: gli Stati Uniti svolgevano, il ruolo di grande importatore finale, indebitandosi e andando in deficit. Tale ruolo è stato storicamente svolto dagli Usa che ha fatto da locomotiva a tutti gli altri paesi. Ma la crisi ha rallentato l'economia americana e gli Stati Uniti hanno smesso essere il grande importatore finale. Ora, il problema è cercare qualcuno che li sostituisca. Per un po' c'è stata la Cina, che è stata il grande importatore dei beni tedeschi e quindi ha trascinato la Germania. La piccola crescita che ha avuto l'Italia è dovuta alla Cina, che importa dalla Germania e noi, essendo sub-fornitori della Germania, veniamo tirati a nostra volta. Ma se la Cina smettesse di comprare tecnologia tedesca, noi, insieme alla Germania, rallenteremmo, come sta avvenedo.

La finanza, oggi, si nasconde dietro allo stato? Questo è un problema politico difficile da sciogliere oggi. Quando si chiede allo stato di intervenire in una crisi economica, siamo abituati a pensare che lo stato sia indipendente dalla finanza. Ma se la finanza condiziona lo stato, qual è il ruolo dello stato in questo capitalismo dominato dalla finanza? La finanza condiziona lo stato e lo stato è al servizio della finanza e delle banche? O lo stato è al servizio dei cittadini? Queste domande si legano a quanto abbiamo già considerato: "sono le banche troppo grosse per fallire (too big to fail)? Lo stato è oggi strettamente legato alle banche; e la crisi delle banche è ormai interconnessa alla crisi dello stato, attraverso la detenzione del debito sovrano.

C'è un libro che cerca di analizzare il comportamento dell'ammi- 
nistrazione Obama durante la crisi e afferma che il comportamento del suo staff economico è stato quello di salvare Wall Street e non di salvare "main street", cioè la gente comune (Sheila Bair, ex capo della Federal Deposit Insurance Corporation, "Bull by the Horns: Fighting to Save Main Street from Wall Street and Wall Street from itself"). Perché? Perché l'amministrazione Obama è dominata dalla finanza. Questo è uno dei problemi politici dell'oggi. Ne è un esempio, in particolare, il ruolo di Summers, l'economista che è stato determinante in molte scelte di Obama, e il ruolo di Geithner, che è il Segretario del Tesoro. Questa non è una storia recente nell'evoluzione del capitalismo finanziario americano. Negli Stati Uniti è una storia che si ripete: tra gli ultimi sei Segretari del Tesoro, ben quattro vengono dalla Goldman Sachs. È molto significativo che un Segretario del Tesoro americano sia una persona che ha ricoperto ruoli fondamentalì nella finanza. Molti dicono: "sì, è perché deve conoscere i mercati ...", altrimenti non potrebbe fare il Segretario del Tesoro. Il problema è veramente complicato pe la democrazia. Clinton ha avuto un grande ruolo nel sostenere questo conflitto di interessi: durante la crisi del Messico del 1994, Robert Rubin, a lungo capo della Goldman Sachs, salva sia la Goldman Sachs che la City Group, che erano coinvolte nella crisi messicana, cioè salva la finanza. E qui mi ricordo il ruolo di Greenspan. Direttore di J.P. Morgan Chase, l'altra grande istituzione finanziaria americana, favorevole alla deregolamentazione, Greenspan diventa presidente della Banca Centrale americana. La stessa cosa accade in Europa e non c'è da stupirsi. Draghi, vice-presidente della Goldman Sachs Europa e incaricato del settore imprese e paesi sovrani, sa molto sulla Grecia. Una delle sue missioni fu quella di vendere prodotti finanziari swap, consentendo la trasformazione del debito sovrano greco. Monti dal 2005 è stato International Advisor per Goldman Sachs. La Goldman Sachs è dovunque ed è occupa i posti dominanti del Tesoro americano, del Tesoro europeo, delle autorità di controllo monetario. Qualcuno dice: "basta banchieri di Wall Street, come Segretari del Tesoro, come banchieri centrali, o come Presidenti del Consiglio".

Vorrei concludere con alcune riflessioni sulla democrazia: abbiamo bisogno di nuove istituzioni; abbiamo bisogno di scelte politiche coscienti, in cui i cittadini capiscano ciò che sta accadendo, senza che qualcun'altro prenda decisioni per loro.

A tale proposito vorrei citare Dani Rodrik, economista americano, un "embedded liberal". Embedded vuol dire un liberale che è anco- 
rato a istituzioni nazionali, ma, attenzione, non un nazionalista. Rodrik è certamente un liberale - com'era Keynes - ed è molto vicino alle posizioni per teoriche in cui le istituzioni devono rispettare il carattere delle nazioni, fin quando le nazioni ci sono (e siamo in una fase storica in cui le nazioni hanno un grande ruolo). Rodrik è critico dei processi di globalizzazione e in particolare di ciò che viene chiamato il Washington Consensus - le direttive imposte dal FMI (Fondo monetario internazionale) ai paesi che si trovano in crisi economica. Secondo Rodrik, c'è la necessità di avere un "policy space", uno spazio per le politiche economiche e sociali, all'interno del quale si possano sviluppare politiche nazionali autonome e non essere costretti a seguire tutti un unico modello di crescita - per esempio, quello tedesco. Ma questo discorso sulla varietà delle politiche nazionali è molto complicato; notate sta anche dietro alla proposta di Keynes a Bretton Woods, il piano Keynes. Nella discussione sugli accordi monetari internazionali di Bretton Woods del 1944 si scontrano due posizioni contrapposte: quella di White, da una parte, che sosteneva la posizione americana e quella di Keynes, dall'altra parte. In questo scontro vincono gli americani e Keynes perde. White difendeva il dollaro come moneta sovrana; Keynes, invece, auspicava una moneta internazionale (il bancor) che non fosse il dollaro: probabilmente Keynes era un antiamericano, ma era anche un difensore di uno "policy space", uno spazio di autonomia nazionale, mentre si stava formulando una riforma istituzionale del sistema monetario internazionale, cioè di un'istituzione che in qualche modo avrebbe gestito la moneta internazionale e gli squilibri commerciali. Nella posizione di Keynes era ben presente il problema di gestire i rapporti tra debitori e creditori. Nella battaglia politca, Keynes perde e vince White: vince il dollaro, che però nel 1971 perde la convertibilità in oro e con questo salta il sistema di Bretton Woods. Dal 1971 in avanti siamo in anarchia, siamo cioè in un "non sistema".

Rodrik riflette su Bretton Woods e sul "non sistema". Il "non sistema" monetario si lega al problema degli squilibri internazionali: è questo il vero anello non visibile nella crisi. In un suo articolo del 2000 e nel libro intitolato Il paradosso della globalizzazione Rodrik pone un "trilemma": possono coesistere globalizzazione, democrazia e stati nazionali? La sua risposta è che ciò sarebbe bellissimo, ma questi tre corni teorici del dilemma, - che poi diventano problemi istituzionali non possono essere soddisfatti contemporaneamente. "Non si possono avere tutti e tre insieme, dobbiamo rinunciare, politicamente e quindi 
economicamente, a uno dei tre". Ci può essere la globalizzazione, la democrazia e una specie di autonomia nazionale (che è la capacità di poter decidere autonomamente; pensiamo solo a quanto sta accadendo in Grecia sulle elezioni)? Si pone di nuovo il problema se gli stati nazionali siano fonte di frizione per i processi di internazionalizzazione dei commerci. Si può avere uno stato nazionale e la globalizzazione? Vi è un problema di democrazia: le decisioni sono prese da altri, ad esempio dal FMI, da organismi del commercio internazionale o dalla UE. Gli stati nazionali sono di fronte a scelte difficili: adottare le regole fissate dai sostenitori del Washington Consensus - e il caso greco è uno di questi (notate che queste imposizioni alla Grecia sono state già imposte al Sud America e ai paesi asiatici nella crisi degli anni '90; quindi, la storia si ripete) - oppure, come afferma Rodrik, "gli Stati devono rinunciare alla loro sovranità per affidarsi a istituzioni internazionali o sovranazionali su un modello federalista". Dev'esserci una scelta esplicita in cui parte della sovranità nazionale viene delegata a livello europeo (il recente scontro fra Hollande e la Merkel è su questo aspetto). Poiché gli stati nazionali sono molto forti, questo appare oggi impossibile politicamente, cioè siamo in una situazione in cui difficilmente i governi nazionali - e la Germania in primis - cedono potere nazionale. Rodrik ritiene che questa cessione di potere nazionale non possa essere una pratica percorribile oggi e nel mondo in cui viviamo. L'unica alternativa possibile, afferma Rodrik, è tornare indietro nel processo di globalizzazione; quindi abbandonare la globalizzazione, "però, attenzione - afferma perderete anche tutti i vantaggi che da essa derivano". Forse dovremmo chiarirci un po' le idee, perché il problema è complicato; e mentre ci chiariamo le idee, il capitalismo si trasforma attraverso nuove crisi. Concludo con un passo di Lunghini che lega strettamente e chiaramente Treasury View, Washington Consensus e Bruxelles consensus:

"La convenzione su cui si è retto sino a ieri l'equilibrio capitalistico, condivisa dai responsabili delle politiche economiche nazionali e internazionali, dai consigli di amministrazione delle banche e delle grandi imprese, e dagli editorialisti più autorevoli, si può riassumere in queste quattro proposizioni:

a. L'economia di mercato è il miglior sistema che si possa concepire: anche grazie alla finanza, essa racchiude in sé le chiavi del progresso materiale e del dinamismo economico e sociale.

b. Perché possa dare tutti i suoi benefici, l'economia di mercato deve essere opportunamente gestita e regolata. 
c. Questa regolazione deve sottostare a due princìpi: condurre una politica monetaria che produca la stabilizzazione dei prezzi; ricercare almeno il pareggio, e se possibile l'attivo, del bilancio pubblico.

d. Privilegiare le riforme strutturali che incentivano il lavoro, riducendo i redditi distribuiti dal sistema di previdenza e assistenza sociale.

A queste quattro proposizioni, ne aggiungo una quinta: la distribuzione del reddito e della ricchezza è il portato dei meriti e dei talenti individuali, e dunque non va modificata, se non a favore dei profitti. ... Al formarsi di questa convenzione, hanno dato un contributo determinante gli economisti ortodossi (tanto gli economisti teorici, quanto gli econometrici)" (G. Lunghini, La Teoria generale e $i$ keynesiani: un'eredità giacente, Accademia nazionale dei Lincei, Gli economisti post-keynesiani di Cambridge e l'Italia, 11 e 12 marzo 2009). 